\title{
METODOLOGIA DE AVALIAÇÃO: ANÁLISE DA QUALIDADE DE APLICATIVOS EDUCACIONAIS PARA MATEMÁTICA DO ENSINO MÉDIO
}

\author{
Monielle Gomes da Silva, IF Fluminense \\ monielle@gmail.com \\ Silvia Cristina Freitas Batista, IF Fluminense \\ silviac@iff.edu.br
}

\begin{abstract}
RESUMO
Muitos aplicativos para dispositivos móveis estão sendo desenvolvidos para matemática. No entanto, é fundamental que esses recursos sejam avaliados, pois nem sempre possuem características adequadas. Nesse sentido, um instrumento que auxilie o professor de matemática na escolha de um aplicativo mais apropriado aos seus objetivos pedagógicos é importante. Assim, este trabalho apresenta uma metodologia de avaliação de qualidade para aplicativos educacionais que permitam o estudo de tópicos matemáticos do Ensino Médio. A metodologia contempla aspectos sobre documentação, usabilidade/questões operacionais, questões pedagógicas e conteúdos matemáticos e permite obter um percentual geral de adequação do aplicativo aos critérios avaliados. Três testes foram promovidos e, por meio destes, a metodologia foi aprimorada até a obtenção de uma versão considerada adequada ao seu propósito.
\end{abstract}

Palavras-chave: metodologia de avaliação, qualidade, aplicativos educacionais, dispositivos móveis, matemática.

\section{EVALUATION METHODOLOGY: QUALITY ANALYSIS OF EDUCATIONAL APPLICATIONS FOR HIGH SCHOOL MATHEMATICS}

\begin{abstract}
Many mobile applications are being developed for Mathematics. However, it is crucial that these resources are evaluated, because they don't always have appropriate characteristics. In this sense, an instrument that helps the Mathematics teacher in choosing an appropriate application to their educational goals is important. This work presents a quality assessment methodology for educational applications that allow the study of High School mathematical topics. The methodology includes aspects of documentation, usability/operational issues, educational issues and mathematical content, and gives an adequacy percentage of the application related to the evaluated criteria. Three tests were promoted and, through them, the methodology has been improved to obtain a version considered to be suitable for its purpose.
\end{abstract}

Keywords: evaluation methodology, quality, educational applications, mobile devices, Mathematics.

\section{Introdução}

A utilização dos dispositivos móveis na educação tem sido estudada em um campo de pesquisa chamado Mobile Learning ou m-learning. Busca-se entender, com as pesquisas dessa área, como as tecnologias móveis podem favorecer a aprendizagem, contribuindo para que a mesma ocorra em qualquer tempo e lugar. Segundo Pachler et al. (2010), 
trata-se de um campo emergente de pesquisa e prática educacional em instituições de ensino e em locais de trabalho e, também, no âmbito na educação informal.

Nesse contexto, o presente trabalho tem como foco os programas educacionais para matemática, desenvolvidos para dispositivos móveis. Tais programas são comumente chamados aplicativos e, portanto, neste artigo, adota-se aplicativo educacional como uma expressão equivalente a software educacional para dispositivos móveis.

Os aplicativos educacionais englobam os, especificamente, desenvolvidos para fins educacionais e, também, aqueles projetados para outros usos, mas que podem ser adaptados para fins pedagógicos, tais como aplicativos para geolocalização, leitores de arquivos, mapas, entre outros (EDUCAUSE, 2010).

Metodologias de avaliação de qualidade específicas para esses aplicativos ainda não são muito comuns. Na pesquisa promovida, foram identificados poucos estudos relacionados à avaliação de aplicativos educacionais, tais como Walker (2010), Boltz e Shoemaker (2010), Schrock (2011) e Amaral (2013). Ressalta-se que avaliar aplicativos por meio de metodologias não específicas implica promover adaptações, pois estes possuem características particulares, como mobilidade, praticidade de uso, manipulação de recursos por meio de toques, adequação ao tamanho das telas, entre outras.

Em particular, considerando a área de matemática, foram identificadas metodologias de avaliação de software, como as propostas por Gladcheff (2001) e Batista (2004). No entanto, não foi encontrada uma metodologia de avaliação específica para aplicativos educacionais dessa área. Assim, este trabalho visa apresentar uma metodologia ${ }^{1}$ para tal fim, tendo por foco aplicativos destinados ao estudo de temas matemáticos do Ensino Médio.

Para atingir o objetivo descrito, abordam-se, na seção 2, aspectos relacionados ao uso pedagógico de dispositivos móveis, de forma geral, e, mais especificamente, na área de matemática. Na seção 3, são descritas algumas propostas para avaliação de aplicativos educacionais, identificadas na literatura. Na seção 4, apresentam-se os procedimentos metodológicos adotados e descrevem-se os três testes realizados, dois com alunos de informática e um com licenciandos em matemática. Finalizando, na seção 5, são tecidas algumas considerações sobre o trabalho realizado.

\section{Dispositivos Móveis na Educação}

As tecnologias móveis tornaram-se parte integrante da sociedade contemporânea, que está cada vez mais digital. Uma pesquisa, divulgada em 2014, mostrou que, em apenas um ano, 20,5 milhões de brasileiros passaram a acessar a Internet por celular ou tablet (F/NAZCA; DATAFOLHA, 2014). O acesso pelo celular, no Brasil, quase dobrou em dois anos, segundo essa pesquisa.

Essa nova sociedade requer uma educação formal que reconsidere a forma de entender o ensino, valorizando práticas e aprendizagens que ocorrem dentro e fora do ambiente escolar, e que amplie os seus horizontes em termos dos recursos que podem contribuir para os desafios atuais (LOBATO; PEDRO, 2012). Segundo Grant e Basye (2014), os dispositivos móveis podem contribuir para mudar o paradigma da sala de aula tradicional, favorecendo uma aprendizagem mais personalizada.

Atividades envolvendo esses dispositivos geralmente apresentam características como interatividade, mobilidade, trabalho em equipe, entre outras (BATISTA et al., 2010). Segundo essas autoras, o desenvolvimento de recursos pedagógicos para tais dispositivos é essencial para a efetiva utilização dos mesmos nas práticas educativas.

Em particular, para matemática, diversos aplicativos estão sendo desenvolvidos. A título de exemplificação, apresentam-se três destes, todos gratuitos: i) TriPlot $3 D$ Graphing Free ${ }^{2}$ : plotador gráfico em 3D, para Android, em inglês, com uma interface 
simples e intuitiva; ii) GeoGebra ${ }^{3}$ : como as demais versões desse programa, a versão para tablets permite trabalhar tópicos matemáticos, de forma dinâmica; esse aplicativo encontra-se disponível em vários idiomas, inclusive o português, e possui versão para Android, iOS e Windows 8; iii) Círculo Unitário Trigonométrico ${ }^{4}$ : aplicativo Android, para estudo de trigonometria, com versão em português e interface simples.

Embora seja possível encontrar uma variedade de aplicativos para matemática, como os mencionados, é importante que avaliações de qualidade sejam realizadas. Entende-se que estas podem contribuir para o desenvolvimento de recursos mais adaptados à proposta de m-learning (BATISTA et al., 2010).

Além dos recursos, diversos estudos também têm sido promovidos associando matemática e m-learning, tais como Marçal et al. (2010), Dias e Araújo Jr. (2012) e Barcelos e Batista (2013). Marçal et al. (2010) identificam os principais requisitos para a elaboração de aplicações m-learning, em especial, aquelas destinadas ao ensino de matemática (requisitos econômicos, socioculturais, pedagógicos, técnicos e relacionados à matemática). Os autores descrevem, ainda, o desenvolvimento de três aplicativos nos quais foram utilizados esses requisitos. $\mathrm{O}$ objetivo dos autores foi fornecer uma base para a criação de um guia prático de desenvolvimento deste tipo de aplicação.

Dias e Araújo Jr. (2012) apresentam um framework conceitual para aplicação de mobile learning no ensino de ciências, tecnologia e matemática, na Educação Básica. A pesquisa envolveu um projeto piloto em matemática, no qual foi utilizado o aplicativo gráfico mePlot Free, em tablets, no Ensino Médio. Os autores observaram que os alunos compreenderam a simplicidade e praticidade da tecnologia móvel (tablet) e, também, seu potencial para interação e relacionamento com os colegas. No geral, os alunos apresentaram alto grau de motivação e satisfação com o uso de tablets.

Barcelos e Batista (2013) realizaram um estudo de caso sobre o uso de aplicativos em tablets na aprendizagem de sistemas lineares. Nessa pesquisa, foi discutida a visão dos licenciandos em matemática sobre os plotadores gráficos, quando utilizados na interpretação geométrica de sistemas lineares $2 \times 2$ e $3 \times 3$. Foram utilizados quatro aplicativos: xGraphing, Calculus Tools, mePlot Free e TriPlot 3D Graphing Free. A experiência promovida permitiu observar a importância da análise de aplicativos para tablets e também da formação adequada de professores para esse propósito.

Observa-se, assim, que a utilização pedagógica de dispositivos móveis tem sido investigada. Entende-se que a utilização de aplicativos de qualidade torna-se fundamental nesse contexto. Considerando essa perspectiva, a seção seguinte aborda a qualidade dos aplicativos educacionais.

\section{Avaliação de Qualidade de Aplicativos Educacionais}

Qualidade de software, de maneira geral, segundo Pressman (2011, p. 724), pode ser definida como "conformidade a requisitos funcionais e de desempenho explicitamente declarados, a padrões de desenvolvimento claramente documentados e a características implícitas que são esperadas de todo software profissionalmente desenvolvido". Existem fatores que podem ser medidos diretamente e os que só podem ser medidos indiretamente, como usabilidade. É necessário comparar o software com alguma informação para chegar a uma indicação de qualidade (PRESSMAN, 2011).

Diversos modelos e normas de qualidade de software têm sido criados (por exemplo, normas ISO, modelo CMM - Capability Maturity Model, modelo MPS.BR Melhoria de Processos do Software Brasileiro). Esses instrumentos servem para medir aspectos relacionados à qualidade do produto e do processo de desenvolvimento e ao nível de maturidade da organização desenvolvedora (MACIEL et al., 2011).

Destaca-se que, durante a elaboração deste trabalho, nenhuma norma ISO específica para aplicativos foi encontrada. No entanto, algumas propostas para avaliação 
de aplicativos educacionais têm sido criadas, dentre as quais as apresentadas por Walker (2010), Boltz e Shoemaker (2010), Schrock (2011) e Amaral (2013), descritas a seguir.

Walker (2010) investigou o impacto do iPod touch no desempenho do aluno. Para tanto, construiu um guia para avaliar a qualidade e a eficácia de um aplicativo, em termos de como o mesmo pode afetar o desempenho do aluno. Seus critérios incluem autenticidade, feedback, diferenciação, facilidade de uso e motivação dos alunos. Foi criado um quadro com uma escala de 1 a 4 e cada item avaliado recebe uma nota, de acordo com os critérios propostos.

Boltz e Shoemaker (2010) refletem sobre a dificuldade de escolher, dentre os diversos aplicativos educacionais disponíveis, aqueles que serão benéficos e mais bem sucedidos em sala de aula. Os autores apresentam algumas recomendações sobre como escolher aplicativos para iPads que melhor atendam às necessidades do professor e dos alunos, tais como: i) ler a descrição dos aplicativos e os comentários de outros usuários; ii) conferir o site do aplicativo e verificar se os desenvolvedores atuam em uma área específica; iii) experimentar o aplicativo antes de utilizá-lo em sala de aula; iv) utilizar o checklist proposto por Walker (2010); v) utilizar o guia de questões iPad App Evaluation Guiding Questions ${ }^{5}$.

Schrock (2011) promove o uso do iPad para apoiar práticas pedagógicas. A autora definiu dois instrumentos de avaliação: um para avaliar os aplicativos usados no iPad e outro para auxiliar no desenvolvimento de aplicativos. Para a avaliação, a autora elaborou um questionário que, inicialmente, coleta dados sobre o aplicativo (título, custo, área, entre outros) e, a seguir, propõe questões destinadas à análise de critérios, tais como navegação, feedback, motivação, entre outros. O questionário possui também um campo para associação do aplicativo a um dos níveis da taxonomia de Bloom e outro para o avaliador informar se recomendaria, ou não, o uso em sala de aula.

No questionário proposto, para auxiliar o desenvolvimento de aplicativos, Schrock (2011) elaborou perguntas em relação à importação de projetos criados em computadores, gravação e inserção de áudio, exportação para outros locais, utilização do touchscreen, entre outros. Schrock (2011) elaborou, também, um checklist baseado na proposta de Walker (2010).

Amaral (2013) faz uma análise de aplicativos matemáticos e estatísticos para tablets. Inicialmente, a autora promoveu uma pesquisa e verificou que, apesar dos tablets serem um fenômeno recente, é possível encontrar boas opções de aplicativos matemáticos, de diversos tipos, gratuitos. Após essa pesquisa, Amaral (2013) analisou alguns aplicativos, levando em consideração uma proposta de avaliação na qual definiu critérios em relação ao custo, disponibilidade, interface e potencial educacional.

Os autores citados apresentam apenas recomendações e propostas com checklist, mas nenhuma metodologia propriamente dita. O presente trabalho baseou-se nessas propostas para desenvolver a metodologia de avaliação de aplicativos. Além disso, foram utilizadas, também, a norma ISO/IEC 9126-16 (ABNT, 2003) e a metodologia SoftMat (BATISTA, 2004). Esta metodologia, apesar de estar relacionada a programas utilizados em desktops, auxiliou na parte pedagógica geral e na específica de matemática, que é o foco de avaliação deste trabalho.

\section{Desenvolvimento da Metodologia de Avaliação}

Nesta seção, inicialmente são apresentados os procedimentos metodológicos adotados no trabalho e, a seguir, descrevem-se o processo de desenvolvimento e os testes da metodologia. 


\subsection{Procedimentos Metodológicos}

Além da identificação e análise de trabalhos que poderiam servir de base para o presente estudo, diversos aplicativos para matemática do Ensino Médio foram experimentados (tais como os descritos na seção 2). Os mesmos foram pesquisados na loja virtual Google Play e manipulados em um tablet com Android, por ser esse o sistema operacional dos tablets do Instituto Federal no qual os testes foram realizados.

Visou-se, com isso, ampliar o conhecimento sobre aplicativos para matemática do Ensino Médio e, assim, identificar aspectos a serem incluídos na metodologia, comparando-os com os já descritos nos trabalhos correlatos identificados.

Tendo por objetivo identificar pontos a serem melhorados, foram promovidos três testes da metodologia elaborada (teste 1, 2 e 3). $\mathrm{O}$ teste 1 foi referente à primeira versão da metodologia e envolveu três participantes: dois alunos do Bacharelado em Sistemas de Informação e uma aluna da Pós-Graduação Lato Sensu de Análise e Gestão de Sistemas de Informação do Instituto Federal considerado, todos com experiência de uso de aplicativos para dispositivos móveis. A versão inicial da metodologia foi enviada, por $e$-mail, para análise dos participantes.

No teste 2, analisou-se a segunda versão da metodologia, obtida a partir das modificações decorrentes do teste 1 . Dessa vez, os avaliadores foram três alunos do Ensino Técnico de Informática Integrado ao Médio do Instituto Federal em questão, participantes de ações promovidas no âmbito de um projeto de pesquisa sobre o uso pedagógico de dispositivos móveis. Os referidos alunos têm trabalhado na seleção e análise de aplicativos educacionais destinados ao estudo de áreas curriculares diversas. Assim, foram considerados adequados para participar do segundo teste da metodologia, que ocorreu no dia 05 de dezembro de 2014. Para a avaliação, cada aluno utilizou um tablet institucional e uma ficha de atividades matemáticas relacionadas ao aplicativo Círculo Unitário Trigonométrico, desenvolvidas especificamente para esse fim.

O teste 3 avaliou a terceira versão e foi realizado em 12 de dezembro de 2014, com seis licenciandos de matemática, três do oitavo período (concluintes do curso), dois do sexto e um do quarto período. Todos com experiência de uso de recursos tecnológicos no processo de ensino e aprendizagem de matemática. Esse teste, portanto, foi promovido com representantes do público alvo da metodologia, uma vez que a mesma é direcionada a professores de matemática (em formação e em serviço).

Para esse terceiro teste, foram selecionados três aplicativos. Para tanto, foi realizado um levantamento na loja virtual Google Play sobre aplicativos direcionados para as seguintes áreas: trigonometria, matrizes e geometria espacial. Esses temas foram escolhidos por serem abordados no Ensino Médio e permitirem contemplar diferentes tipos de aplicativos. O tema matrizes, em geral, é estudado sob um enfoque mais algébrico. $\mathrm{O}$ estudo de trigonometria envolve análises algébricas e gráficas e a geometria espacial trata dos métodos para o estudo de objetos espaciais e das relações entre elementos desses objetos. Na área de Trigonometria, foi selecionado o Círculo Unitário Trigonométrico, que continha, na época, 443 avaliações na loja Google Play, com nota 4,5 (em um máximo de cinco). Em Geometria, considerando a geometria espacial, o GeoGebra foi selecionado. Esse aplicativo possuía 4.281 avaliações, com nota 3,9. Para matrizes, foi selecionado o aplicativo Calculadora Matriz, com 87 avaliações e nota 3,2. Buscou-se, com isso, identificar aplicativos com características e avaliações de qualidade diferentes, de forma a possibilitar melhor análise dos aspectos contemplados na metodologia.

No teste 3, foram utilizados tablets institucionais e atividades matemáticas investigativas sobre os aplicativos, para facilitar o reconhecimento dos mesmos.

A seguir, descreve-se o desenvolvimento das versões da metodologia. 


\subsection{Primeira Versão}

A partir das pesquisas e estudos promovidos, foi estruturada a primeira versão da metodologia. Foram elaboradas afirmativas relacionadas aos seguintes critérios: i) documentação; ii) usabilidade/questões operacionais; iii) questões pedagógicas; iv) conteúdos matemáticos. Diante de cada uma delas, o avaliador deve escolher uma das opções: Não se Aplica (NA), Discordo Completamente (DC), Discordo (D), Não Concordo Nem Discordo (NCND), Concordo (C) e Concordo Completamente (CC). A opção NA deve ser assinalada quando a afirmativa analisada não for coerente com o aplicativo avaliado. Assim, a opção NA não tem o mesmo significado de NCND, sendo essa última entendida como uma alternativa para um avaliador que se manteve neutro sobre um determinado critério coerente com o aplicativo.

Os trabalhos relacionados foram fundamentais para a elaboração das afirmativas. Destacam-se algumas dessas contribuições, identificando os critérios em que cada trabalho mais colaborou: i) Walker (2010) - facilidade de uso e motivação dos alunos; ii) Boltz e Shoemaker (2010) - documentação; iii) Schrock (2011) - usabilidade e feedback; iv) Amaral (2013) - interface e potencial educacional; v) Batista (2004) critérios pedagógicos e matemáticos; vi) norma ISO/IEC 9126-1 (ABNT, 2003) funcionalidade, usabilidade, confiabilidade e portabilidade.

A versão inicial da metodologia foi enviada aos três participantes do primeiro teste, solicitando que os mesmos analisassem as afirmativas elaboradas e, caso necessário, sugerissem alterações. Dois participantes não apresentaram sugestões, apenas mencionaram que as afirmativas estavam adequadas. $\mathrm{O}$ terceiro registrou que todas as afirmativas propostas eram importantes, porém algumas em maior grau do que outras. Esse comentário alertou para necessidade da metodologia possibilitar a inserção, por parte do usuário, de peso para cada afirmativa, de acordo com sua visão. Após esse teste, teve início a elaboração da segunda versão da metodologia.

\subsection{Segunda Versão}

A partir do reconhecimento da importância da possibilidade de inserção de pesos diferenciados, observou-se que a metodologia precisaria ter, também, um mecanismo que permitisse o cálculo de um percentual de adequação do aplicativo segundo os critérios avaliados. Assim:

- foram atribuídos valores às opções, da seguinte forma: i) NA: não recebe pontuação; ii) CC: 1 ; iii) C: 0,75 ; iv) NC/ND: 0,5; v) D: 0,25; vi) DC: 0;

- foi criada uma coluna para que o usuário possa atribuir um peso a cada uma das afirmativas. A escala varia de 1 a 3 , na qual 1 é o peso para afirmativas menos importantes e 3 para as consideradas muito importantes. Por padrão, todas as afirmativas já têm peso 1, cabendo ao usuário alterar para 2 ou 3, caso deseje;

- para o cálculo do resultado final (percentual de adequação do aplicativo), foi implementada a fórmula mostrada na figura 1 (já considerando as 30 afirmativas da versão final da metodologia). Na mesma, $R_{f}$ é o resultado final, $A_{n}$ é o valor correspondente à opção assinalada pelo usuário na afirmativa $n$ e $P_{n}$ é o peso da afirmativa $n$. Esclarece-se que se a opção NA for selecionada, o peso da afirmativa recebe 0 (zero), independentemente do valor exibido na coluna de pesos.

$$
R_{f}=\frac{\sum_{n=1}^{30} A_{n} P_{n}}{\sum_{n=1}^{30} P_{n}}
$$

Figura 1 - Fórmula do percentual de adequação 
Tendo elaborada essa segunda versão, foi realizado o teste 2, no qual foi promovida a avaliação de um aplicativo de matemática. Para tanto, os participantes realizaram atividades para reconhecimento dos recursos e, a seguir, utilizaram a metodologia para promover a avaliação. Tendo em vista o objetivo do teste, os participantes foram orientados a manter a atenção na análise da metodologia. O reconhecimento das funcionalidades do aplicativo e a avaliação do mesmo foram ações destinadas apenas a possibilitar melhor análise das afirmativas propostas na metodologia. A partir dos dados levantados, foi organizada a terceira versão da metodologia.

\subsection{Terceira Versão}

Descrevem-se, a seguir, algumas sugestões dadas pelos participantes do teste 2 e as modificações decorrentes das mesmas. Para facilitar a descrição, os alunos foram nomeados A1, A2 e A3. O aluno A1 não apresentou sugestões.

- A2 e A3 sugeriram a reformulação da afirmativa "permite que o aluno utilize independentemente, sem a necessidade do professor", pertencente ao bloco Pedagógico, para que a mesma ficasse mais fácil de ser entendida. A afirmativa foi reformulada para "permite que o aluno realize atividade extraclasse, de forma independente";

- A2 sugeriu a inclusão de uma afirmativa sobre tutorial/ajuda dentro do aplicativo. A afirmativa "possui tutorial interno sobre sua utilização" foi inserida no bloco Documentação;

- A3 recomendou que na afirmativa "possui mecanismo de feedback para auxiliar o usuário em caso de erro", do bloco Pedagógico, fosse verificada se a utilização da palavra feedback não dificultaria o entendimento. Nesse sentido, a afirmativa foi alterada para "possui mecanismo de feedback (resposta, retorno) para auxiliar o usuário em caso de erro".

Após a promoção das modificações mencionadas, foi realizado o teste final (teste 3) do qual resultou a quarta versão da metodologia (versão final).

\subsection{Quarta Versão}

Para análise dos dados do teste 3 , os licenciandos foram nomeados L1, L2, ..., L6. Descrevem-se, a seguir, algumas das sugestões apresentadas e as respectivas alterações.

- L1, L2 e L3 sugeriram que a palavra "plataformas" da afirmativa "possui versão para diversas plataformas", do bloco Usabilidade/Questões Operacionais, fosse substituída por outra de mais fácil compreensão para aqueles que não são da área de tecnologia. A palavra foi substituída pela expressão "sistemas operacionais";

- L2 recomendou a inclusão de uma afirmativa sobre as propagandas e anúncios que aparecem durante a utilização de alguns aplicativos. A afirmativa "não apresenta propagandas/anúncios durante a execução" foi adicionada ao bloco Usabilidade/Questões Operacionais;

- L3 sugeriu a modificação da palavra "isento" na afirmativa "esteve isento de falhas durante sua utilização", do bloco Usabilidade/Questões Operacionais, justificando que qualquer aplicativo pode falhar. A sugestão foi analisada, mas como o usuário tem uma escala para classificar o quanto concorda com a afirmativa, a mesma não foi alterada;

- L5 propôs que a afirmativa "permite a utilização de touchscreen", do bloco Usabilidade/Questões Operacionais, fosse reelaborada. A sugestão foi avaliada e a afirmativa foi excluída; 
- L5 e L6 sugeriram que a afirmativa "permite sua execução a partir do cartão de memória", do bloco Usabilidade/Questões Operacionais, fosse revista. A sugestão foi avaliada e a afirmativa mantida. Embora muitos dispositivos móveis já possuam um espaço considerável na memória interna, outros utilizam o cartão SD para expandir o espaço, assim, entende-se que o aplicativo deve permitir sua execução por meio deste tipo de memória.

Destaca-se que os participantes dos testes, de maneira geral, contribuíram significativamente para a obtenção da versão final da metodologia, por meio das várias sugestões dadas.

No teste 3, ao analisar as avaliações de um mesmo aplicativo, foi possível observar que os percentuais de adequação obtidos foram, de maneira geral, próximos entre si. Como os mesmos foram obtidos em um teste da metodologia, optou-se por não apresentá-los, pois poderiam sofrer alterações após os ajustes promovidos. No entanto, destaca-se que os mesmos foram totalmente coerentes com as análises prévias dos aplicativos, promovidas pelas pesquisadoras.

A quarta versão (Figura 2), obtida após as considerações do teste 3, foi analisada pelas pesquisadoras e considerada adequada ao seu propósito de auxiliar o professor de matemática na escolha de um aplicativo para ser utilizado no Ensino Médio. A metodologia contém, além das 30 afirmativas a serem avaliadas, um campo inicial para informações básicas e outro final para eventuais comentários do avaliador.

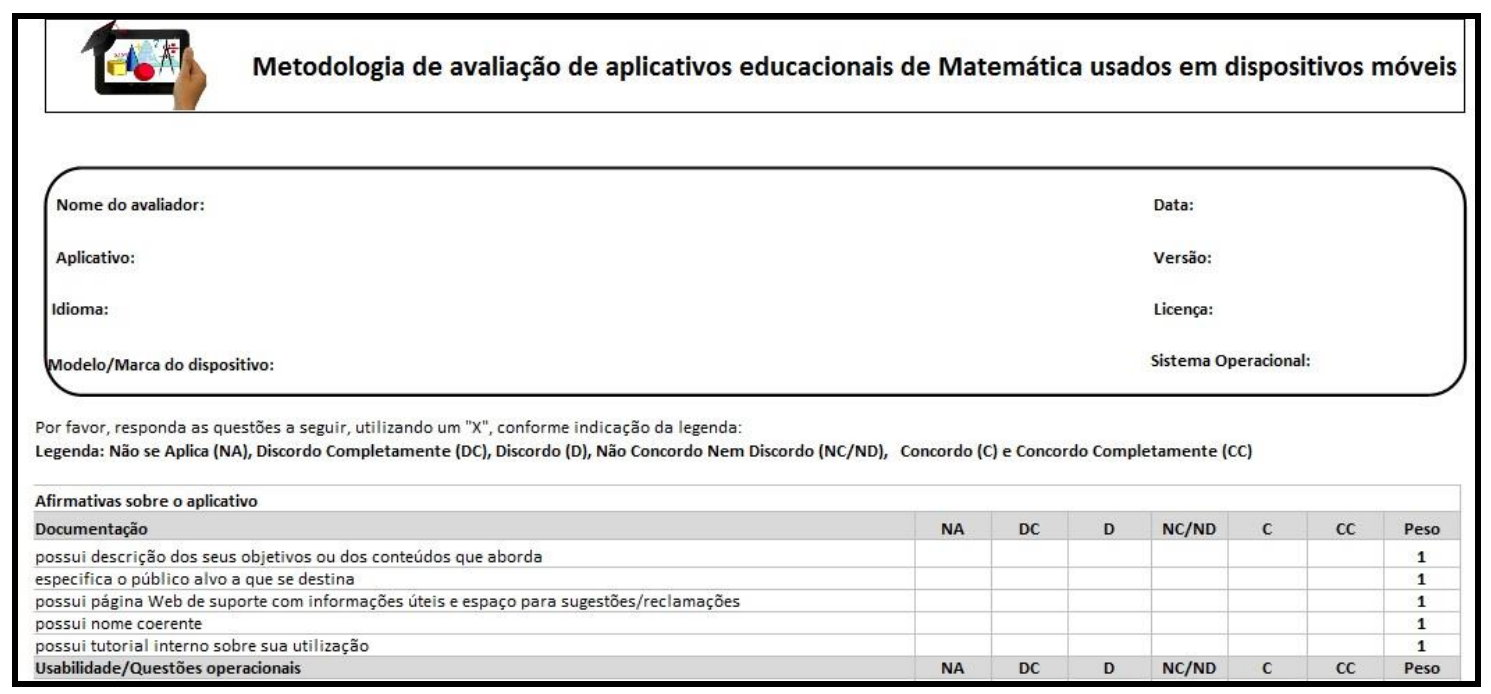

Figura 2 - Metodologia desenvolvida

\section{Considerações Finais}

Existem, atualmente, diversos aplicativos para a utilização em dispositivos móveis, destinados à educação. Particularmente, na área de matemática, esses aplicativos podem auxiliar no processo de aprendizagem, facilitando a compreensão de conteúdos.

Por meio do levantamento realizado no Google Play, vários aplicativos gratuitos para trigonometria, geometria espacial e matrizes foram identificados. No entanto, foi possível observar que alguns: i) apresentam somente fórmulas; ii) apenas realizam cálculos, sem favorecer a realização de atividades investigativas; iii) contemplam partes muito específicas de um conteúdo, não possibilitando estudos mais abrangentes; iv) apresentam recursos que não funcionam como esperado. Assim, observou-se que, dependendo do objetivo pedagógico do professor, a seleção de um aplicativo pode não ser tarefa simples, apesar da quantidade de recursos identificada numa busca. Ressaltase que as avaliações de outros usuários, disponíveis no Google Play, são muito úteis, 
mas, não substituem uma avaliação pessoal do professor, pois é fundamental considerar os propósitos educacionais a serem alcançados.

O panorama descrito evidencia a importância da proposta deste trabalho, que foi desenvolver uma metodologia para avaliação da qualidade de aplicativos para matemática do Ensino Médio. Objetivou-se disponibilizar um recurso para auxiliar os professores na busca de aplicativos mais adequados para suas ações pedagógicas.

$\mathrm{Na}$ metodologia, visou-se contemplar critérios considerados essenciais. Certamente, outros aspectos poderiam ser incluídos, porém isso poderia comprometer a praticidade de uso, ao torná-la cansativa. Destaca-se que a metodologia pode ser adaptada para avaliar aplicativos destinados ao Ensino Superior e, também, para outras disciplinas, bastando para isso alterar o bloco Conteúdos Matemáticos.

Como estudos futuros, pretende-se experimentar a metodologia com professores em serviço. Visa-se, também, disponibilizá-la de forma que o professor possa responder às afirmativas diretamente em uma página Web (atualmente, a metodologia encontra-se em uma planilha eletrônica, cujo arquivo está disponível na Internet). Além disso, pretende-se realizar estudos em relação ao desenvolvimento de aplicativos educacionais considerando os requisitos levantados nesse trabalho.

\section{Notas de Texto}

${ }^{1}$ Disponível em: <https://sites.google.com/site/monielle/metodologia-de-avaliacao-de-aplicativos $>$.

${ }^{2}$ Disponível em: $<$ https://play.google.com/store/apps/details?id=com.seriocon.triplotfree $>$.

${ }^{3}$ Disponível em: <http://www.geogebra.org/download>.

${ }^{4}$ Disponível em:

<https://play.google.com/store/apps/details?id=processing.test.trigonometrycircleandroid $>$.

${ }^{5}<$ https://ipad2educate.wikispaces.com/file/view/iPad+Evaluation+Questions.pdf>.

${ }^{6}$ Embora utilizada no presente trabalho, a norma ISO/IEC 9126-1 foi cancelada em 2013. As normas ISO/IEC 25000 representam a evolução de diversas outras normas, em particular das ISO/IEC 9126.

\section{Referências Bibliográficas}

ABNT - ASSOCIAÇÃO BRASILEIRA DE NORNAS TÉCNICAS. Engenharia de software - Qualidade de produto: NBR ISO/IEC 9126-1: 2003.

AMARAL, P. G. R. Softwares matemáticos e estatísticos para tablets: uma primeira análise. Dissertação (Mestrado Profissional em Matemática em Rede Nacional PROFMAT). Universidade Federal Fluminense, Rio de Janeiro, 2013.

BARCELOS, G. T.; BATISTA, S. C. F. Uso de Aplicativos em Tablets no Estudo de Sistemas Lineares: percepção de licenciandos em matemática. In: CONFERÊNCIA INTERNACIONAL SOBRE INFORMÁTICA NA EDUCAÇÃO - TISE, 23., 2013, Porto Alegre. Anais. Porto Alegre, RS: PUCRS/ Universidad de Chile, 2013. p. 168175.

BATISTA, S. C. F. SoftMat: um repositório de softwares para matemática do Ensino Médio - um instrumento em prol de posturas mais conscientes na seleção de softwares Educacionais. Dissertação (Mestrado em Ciências de Engenharia). Campos dos Goytacazes, RJ, Universidade Estadual do Norte Fluminense - UENF, 2004.

BATISTA, S. C. F.; BEHAR, P. A.; PASSERINO, L. M. Recursos Pedagógicos para dispositivos Móveis: uma análise com foco na matemática. Revista Novas Tecnologias na Educação (RENOTE), v. 8, n. 3, p.1-10, 2010.

BOLTZ, B.; SHOEMAKER, A. iPad App Evaluation Guiding Questions Education App. 2010. Disponível em: <https://ipad2educate.wikispaces.com/App+Evaluation>. Acesso em: 20 abr. 2015. 
DIAS, E. J.; ARAÚJO JR., C. F. Mobile Learning no Ensino de Matemática: um framework conceitual para uso dos tablets na educação básica. In: ENCONTRO DE PRODUÇÃO DISCENTE PUCSP/CRUZEIRO DO SUL. 2012, São Paulo. Anais. São Paulo, 2012, p. 1-13. Disponível em: <http://revistapos.cruzeirodosul.edu.br/index.php/epd/article/viewFile/485/406>.

Acesso em: 21 abr. 2015.

EDUCAUSE. 7 things you should know about Mobile Apps for Learning. 2010. Disponível em: <http://net.educause.edu/ir/library/pdf/ELI7060.pdf>. Acesso em: 22 abr. 2015.

F/NAZCA; DATAFOLHA. Internet móvel, cidadania e consumo no Brasil. 2014. Disponível em: <http://www.fnazca.com.br/wp-content/uploads/2014/12/fradar14_publica-site.pdf $>$. Acesso em: 23 abr. 2015.

GLADCHEFF, A. P. Um instrumento de avaliação da qualidade para software educacional de Matemática. 2001. Dissertação (Mestrado em Ciência da Computação) - Instituto de Matemática e Estatística da USP/São Paulo, 2001. Disponível em: <http://www.lbd.dcc.ufmg.br/colecoes/rbie/10/1/007.pdf>. Acesso em: 20 abr. 2015.

GRANT, P.; BASYE, D. Personalized learning: a guide for engaging students with technology. International Society for Technology in Education. e-book. 2014. Disponível em: <http://www.iste.org/handlers/ProductAttachment.ashx?ProductID=3122\&Type=Downl oad>. Acesso em: 23 abr. 2015.

LOBATO, A.; PEDRO, N. As tecnologias móveis no processo de ensino e aprendizagem da língua inglesa: Um estudo exploratório no CENFIC. In: CONGRESSO INTERNACIONAL TIC E EDUCAÇÃO, 2., 2012, Lisboa. Anais. Lisboa, Portugal, 2012, p. 318-333. Disponível em: <http://ticeduca.ie.ul.pt/atas/pdf/242.pdf>. Acesso em: 22 abr. 2015.

MACIEL, A. C. F.; VALLS, C.; SAVOINE, M. M. Análise da qualidade de software utilizando as normas 12207, 15504, ISO 9000-3 e os modelos CMM/CMMI e MPS.BR. Revista Cientifica do ITPAC, v. 4, p. 1-13, 2011. Disponível em: <www.itpac.br/arquivos/Revista/44/5.pdf>. Acesso em: 21 abr. 2015.

MARÇAL, E.; LIMA, L.; MELO JÚNIOR; VIANA, W.; ANDRADE, R.; RIBEIRO, J. W. Da Elicitação de Requisitos ao Desenvolvimento de Aplicações de Mobile Learning em Matemática. In: SIMPÓSIO BRASILEIRO DE INFORMÁTICA NA EDUCAÇÃO (SBIE), 21., 2010, João Pessoa. Anais. João Pessoa, PB, 2010, p. 1-11. Disponível em: <http://www.lbd.dcc.ufmg.br/colecoes/sbie/2010/0028.pdf>. Acesso em: 20 abr. 2015.

PACHLER, N.; BACHMAIR, B.; COOK, J. Mobile Learning: Structures, Agency, Practices. New York: Springer, 2010.

PRESSMAN, R. S. Engenharia de Software. São Paulo: Pearson, 2011.

SCHROCK, K. Critical Evaluation of a Content-Based mobile apps. 2011. Disponível em: <http://www.ipads4teaching.net/critical-eval-of-apps.html>. Acesso em: 21 abr. 2015.

WALKER, H. Evaluation Rubric for IPod Apps. 2010. Disponível em: $<$ http://learninginhand.com/static/50eca855e4b0939ae8bb12d9/50ecb58ee4b0b16f176a 9e7d/50ecb595e4b0b16f176aaab8/1288148200553/AppRubric.pdf>. Acesso em: 21 de abr. 2015. 